\title{
Security Amplification for Interactive Cryptographic Primitives
}

\author{
Yevgeniy Dodis ${ }^{1}$, Russell Impagliazzo ${ }^{2}$, Ragesh Jaiswal ${ }^{3}$, and Valentine Kabanets ${ }^{4}$ \\ ${ }^{1}$ New York University \\ dodis@cs. nyu .edu \\ ${ }^{2}$ University of California at San Diego and IAS \\ russell@cs.ucsd.edu \\ ${ }^{3}$ Columbia University \\ rjaiswal@cs.columbia.edu \\ ${ }^{4}$ Simon Fraser University \\ kabanets@cs.sfu.ca
}

\begin{abstract}
Security amplification is an important problem in Cryptography: starting with a "weakly secure" variant of some cryptographic primitive, the goal is to build a "strongly secure" variant of the same primitive. This question has been successfully studied for a variety of important cryptographic primitives, such as one-way functions, collision-resistant hash functions, encryption schemes and weakly verifiable puzzles. However, all these tasks were non-interactive. In this work we study security amplification of interactive cryptographic primitives, such as message authentication codes (MACs), digital signatures (SIGs) and pseudorandom functions (PRFs). In particular, we prove direct product theorems for MACs/SIGs and an XOR lemma for PRFs, therefore obtaining nearly optimal security amplification for these primitives.

Our main technical result is a new Chernoff-type theorem for what we call Dynamic Weakly Verifiable Puzzles, which is a generalization of ordinary Weakly Verifiable Puzzles which we introduce in this paper.
\end{abstract}

\section{Introduction}

Security amplification is a fundamental cryptographic problem: given a construction $C$ of some primitive $P$ which is only "weakly secure", can one build a "strongly secure" construction $C^{\prime}$ from $C$ ? The first result in this domain is a classical conversion from weak one-way functions to strong one-way function by Yao [Yao82] (see also [Gol01]): if a function $f$ is only mildly hard to invert on a random input $x$, then, for appropriately chosen $n$, the function $F\left(x_{1}, \ldots, x_{n}\right)=\left(f\left(x_{1}\right), \ldots, f\left(x_{n}\right)\right)$ is very hard to invert. The above result is an example of what is called the direct product theorem, which, when true, roughly asserts that simultaneously solving many independent repetitions of a mildly hard task is a much harder "combined task". Since the result of Yao, such direct product theorems have have been successfully used to argue security amplification of several other important cryptographic primitives, such as collision-resistant hash functions [ $\mathrm{CRS}^{+}$07], encryption schemes [DNR04] and weakly verifiable puzzles [CHSO5, [JK08].

The original version of this chapter was revised: The copyright line was incorrect. This has been corrected. The Erratum to this chapter is available at DOI: 10.1007/978-3-642-00457-5_36

O. Reingold (Ed.): TCC 2009, LNCS 5444, pp. 128-145,2009.

(C) Springer-Verlag Berlin Heidelberg 2009 
However, all the examples above are non-interactive: namely, after receiving its challenge, the attacker needs to break the corresponding primitives without any further help or interaction. This restriction turns out to be important, as security amplification, and, in particular, direct product theorems become much more subtle for interactive primitives. For example, Bellare, Impagliazzo and Naor [BIN97] demonstrated that that parallel repetition does not, in general, reduce the soundness error of multiround (computationally sound) protocols, and this result was further strengthened by Pietrzak and Wikstrom [PW07]. On the positive side, parallel repetition is known to work for the special case of three-round protocols [BIN97] and constant-round publiccoin protocols [PV07]. However, considerably less work has been done in the security amplification of more "basic" cryptographic primitives requiring interaction, such as block ciphers, message authentications codes (MACs), digital signatures (SIGs) and pseudorandom functions (PRFs). For example, Luby and Rackoff [LR86] (see also [NR99. Mye99]) showed how to improve the security of a constant number of pseudorandom permutation generators by composition, while Myers [Mye03] showed that a (non-standard) variant of the XOR lemma [Yao82, Lev87, Imp95, GNW95] holds for PRFs. In particular, the known results for the interactive case are either weaker or more specialized than those for the non-interactive case. The difficulty is that, for instance in the case of MACs, the attacker has oracle access to the corresponding "signing" and "verification" oracles, and the existing techniques do not appear to handle such cases.

In this work we study the question of security amplification of MACs, SIGs and PRFs, showing how to convert a corresponding weak primitive into a strong primitive. In brief, we prove a direct product theorem for MACs/SIGs (and even a Chernofftype theorem to handle MACs/SIGs with imperfect completeness), and a (regular) XOR lemma for PRFs. Before describing these results in more details, however, it is useful to introduce our main technical tool for all these cases - a Chernoff-type theorem for what we call Dynamic Weakly Verifiable Puzzles (DWVPs) - which is of independent interest.

Dynamic Weakly Verifiable Puzzles. Recall, (non-dynamic) weakly verifiable puzzles (WVPs) were introduced by Canetti, Halevi and Steiner [CHSO5] to capture the class of puzzles whose solutions can only be verified efficiently by the party generating the instance of the puzzle. This notion includes, as special cases, most previously mentioned non-interactive primitives, such as one-way functions, collision-resistant hash functions, one-way encryption schemes, CAPTCHAs, etc. To handle also interactive primitives, such as MACs and SIGs (and also be useful later for PRFs), in Section [3 we generalize this notion to that of dynamic WVPs (DWVPs) as follows. Just like in WVPs, one samples a pair $(x, \alpha)$ from some distribution $\mathcal{D}$, where $\alpha$ is the secret advice used to verify proposed solutions $r$ to the puzzle $x$. Unlike WVPs, however, each $x$ actually defines a set of related puzzles, indexed by some value $q \in Q$, as opposed to a single puzzle (which corresponds to $|Q|=1$ ). An efficient verification algorithm $R$ for the DWVP uses $\alpha$ and the puzzle index $q$ to test if a given solution $r$ is correct. An attacker $B$ has oracle access to this verification procedure. Additionally, the attacker has oracle access to the hint oracle: given an index $q$, the hint oracle returns some hint value $H(\alpha, q)$, presumably "helping" the attacker to solve the puzzle $q$. The attacker wins the DWVP game if it ever causes the verification oracle to succeed on a query $q \in Q$ 
not previously queried to the hint oracle. As we see, this abstraction clearly includes MACs and SIGs as special cases. It also generalizes ordinary WVPs, corresponding to $|Q|=1$. We say that the DWVP is $\delta$-hard, if no (appropriately bounded) attacker can win the above game with probability more than $(1-\delta)$.

Our main technical result is the following (informally stated) Chernoff-type theorem for DWVPs. Given $n$ independently chosen $\delta$-hard DWVPs on some index set $Q$, the chance of solving more than $(n-(1-\gamma) \delta n)$ DWVPs - on the same value $q \in Q$ and using less than $h$ "hint" queries $q^{\prime} \neq q$ - is proportional to $h \cdot e^{-\Omega\left(\gamma^{2} \delta n\right)}$; the exact statement is given in Theorem 3 . Notice, the value $0<\gamma \leq 1$ measures the "slackness parameter". In particular, $\gamma=1$ corresponds to the direct product theorem where the attacker must solve all $n$ puzzles (on the same $q$ ). However, setting $\gamma<1$ allows to handle the setting where even the "legitimate" users, - who have an advantage over the attacker, like knowing $\alpha$ or being humans, - can also fail to solve the puzzle with some probability slightly less than $(1-\gamma) \delta$.

This result generalizes the corresponding Chernoff-type theorem of Impagliazzo, Jaiswal and Kabanets [IJK08] for standard, non-dynamic, WVPs. However, the new theorem involves a considerably more complicated proof. The extra difficulties are explained in Section 3.1. In essence, in order to amplify security, the attacker $B$ for the single DWVP must typically execute the assumed attacker $A$ for the "threshold" variant several times, before obtaining sufficient "confidence" in the quality of the solutions output by $A$. In each of these "auxiliary" runs, however, there is a chance that $A$ will ask a hint query for the index $q$ which is equal to the one that $A$ is going to solve in the "actual" run leading to the forgery, making $B$ 's forgery value $q$ "old". Thus, a new delicate argument has to be made to argue security in this scenario. At a high level, the argument is somewhat similar to Coron's improved analysis [Cor00] of the full domain hash signature scheme, although the details differ. See Theorem 3 for the details.

Applications to MACs, SIGs and PRFs. Our main technical result above almost immediately implies security amplification for MACs and SIGs, even with imperfect completeness. For completeness, we briefly state the (asymptotic) result for the MAC case. (The case of SIGs and the exact security version for both cases are immediate.) We assume that the reader is familiar with the basic syntax and the standard Chosen Message Attack (CMA) scenario for a MAC, which is given by a tagging algorithm Tag and the verification algorithm Ver. We denote the secret key by $s$, and allow the tagging algorithm to be probabilistic (but not stateful). Given the security parameter $k$, we say that the MAC has completeness error $\beta=\beta(k)$ and unforgeability $\delta=\delta(k)$, where $\beta<\delta$, if for any message $m, \operatorname{Pr}(\operatorname{Ver}(s, m, \operatorname{Tag}(s, m))=1) \geq 1-\beta$, and that no probabilistic polynomial-time attacker $B$ can forge a valid tag for a "fresh" message $m$ with probability greater than $(1-\delta)$ during the CMA attack.

The MAC $\Pi$ is said to be weak if $\delta(k)-\beta(k) \geq 1 / \operatorname{poly}(k)$, for some polynomial poly, and is said to be strong if, for sufficiently large $k, \beta(k) \leq \operatorname{negl}(k)$ and $\delta(k) \geq$ $1-\operatorname{negl}(k)$, where $\operatorname{negl}(k)$ is some negligible function of $k$. Given an integer $n$ and a number $\gamma>0$, we can define the "threshold direct product" MAC $\Pi^{n}$ in the natural way: the key of $\Pi^{n}$ consists of $n$ independent keys for the basic MAC, the tag of $m$ contains the concatenation of all $n$ individual tags of $m$, and the verification accepts an 
$n$-tuples of individual tags if at least $(n-(1-\gamma) \delta n)$ individual tags are correct. Then, a straightforward application of Theorem 3 gives:

Theorem 1. Assume $\Pi$ is a weak MAC. Then one can choose $n=\operatorname{poly}(k)$ and $\gamma>0$ so that $\Pi^{n}$ has completeness error $2^{-\Omega(k)}$ and unforgeability $\left(1-2^{-\Omega(k)}\right)$. In particular, $\Pi^{n}$ is a strong MAC.

We then use our direct product result for MACs to argue the XOR lemma for the security amplification of PRFs. Namely, in Section 4.2 we show that the XOR of several independent weak PRFs results in a strong PRF (see Section 4.2 for definitions). It is interesting to compare this result with a related XOR lemma for PRFs by Myers [Mye03]. Meyers observed that the natural XOR lemma above cannot hold for $\delta$-pseudorandom PRFs, where $\delta \geq \frac{1}{2}$. In particular, a PRF one of whose output bits is a constant for some input can potentially reach security (almost) $1 / 2$, but can never be amplified by a simple XOR. Because of this counter-example, Meyers had a more complicated XOR lemma for PRFs, where a separate pad was selected for each $\delta$-pseudorandom PRF, and showed that this variant worked for any $\delta<1$. In this work, we show that Meyers' counter-example is the worst: the simple XOR lemma holds for $\delta$-pseudorandom PRFs, for any $\delta<\frac{1}{2}$.

The PRF result generally follows the usual connection between the direct product theorems and the XOR lemmas first observed by [GNW95], but with a subtlety. First, it is easy to see that it suffices to consider Boolean PRFs. For those, we notice that a $\delta$ pseudorandom PRF is also a $(1-2 \delta)$-unforgeable (Boolean) MAC (this is where $\delta<\frac{1}{2}$ comes in). Then, we apply the direct product theorem to obtain a strong (non-Boolean) MAC. At this stage, one typically applies the Goldreich-Levin [GL89] theorem to argue that the XOR of a random subset of (strong) MACs is a PRF. Unfortunately, as observed by Naor and Reingold [NR98], the standard GL theorem does not work in general for converting unpredictability into pseudorandomness, at least when the subset is public (which will ultimately happen in our case). However, [NR98] showed that the conversion does work when $r$ is kept secret. Luckily, by symmetry, it is easy to argue that for "direct product MACs", keeping $r$ secret or public does not make much difference. Indeed, by slightly adjusting the analysis of [NR98] to our setting, we will directly obtain the desired XOR lemma for PRFs.

Finally, in Section 4.1 we observe a simple result regarding the security amplification of pseudorandom generators (PRGs). This result does not use any new techniques (such as our Chernoff-type theorem). However, we state it for completeness, since it naturally leads to the (more complicated) case of PRFs in Section 4.2 and, as far as we know, it has never explicitly appeared in the literature before.

\section{Preliminaries}

For a natural number $k$, we will denote by $[k]$ the set $\{1, \ldots, k\}$.

Lemma 1 (Hoeffding bound). Let $X_{1}, \ldots, X_{t}$ be independent identically distributed random variables taking values in the interval $[0,1]$, with expectation $\mu$. Let $\chi=$ $(1 / t) \sum_{i=1}^{t} X_{i}$. For any $0<\nu \leq 1$, we have $\operatorname{Pr}[\chi<(1-\nu) \mu]<e^{-\nu^{2} \mu t / 2}$. 
Theorem 2 ([GL89]). There is a probabilistic algorithm Dec with the following property. Let $a \in\{0,1\}^{k}$ be any string, and let $O:\{0,1\}^{k} \rightarrow\{0,1\}$ be any predicate such that $\left|\operatorname{Pr}_{z \in\{0,1\}^{k}}[O(z)=\langle a, z\rangle]-1 / 2\right| \geq \nu$ for some $\nu>0$. Then, given $\nu$ and oracle access to the predicate $O$, the algorithm Dec runs in time $\operatorname{poly}(k, 1 / \nu)$, and outputs $a$ list of size $O\left(1 / \nu^{2}\right)$ such that, with probability at least $3 / 4$, the string $a$ is on the list.

\subsection{Samplers}

We will consider bipartite graphs $G=G(L \cup R, E)$ defined on a bipartition $L \cup R$ of vertices; we think of $L$ as left vertices, and $R$ as right vertices of the graph $G$. We allow graphs with multiple edges. For a vertex $v$ of $G$, we denote by $N_{G}(v)$ the multiset of its neighbors in $G$; if the graph $G$ is clear from the context, we will drop the subscript and simply write $N(v)$. Also, for a vertex $x$ of $G$, we denote by $E_{x}$ the set of all edges in $G$ that are incident to $x$. We say that $G$ is bi-regular if the degrees of vertices in $L$ are the same, and the degrees of vertices in $R$ are the same.

Let $G=G(L \cup R, E)$ be any bi-regular bipartite graph. For a function $\lambda:[0,1] \times$ $[0,1] \rightarrow[0,1]$, we say that $G$ is a $\lambda$-sampler if, for every function $F: L \rightarrow[0,1]$ with the average value $\operatorname{Exp}_{x \in L}[F(x)] \geq \mu$ and any $0<\nu<1$, there are at most $\lambda(\mu, \nu) \cdot|R|$ vertices $r \in R$ where $\operatorname{Exp}_{y \in N(r)}[F(y)] \leq(1-\nu) \mu$.

We will use the following properties of samplers (proved in [IJKW08, [JK08]). The first property says that for any two large vertex subsets $W$ and $F$ of a sampler, the fraction of edges between $W$ and $F$ is close to the product of the densities of $W$ and $F$.

Lemma 2 ([|⿱一JUKWW08]). Suppose $G=G(L \cup R, E)$ is a $\lambda$-sampler. Let $W \subseteq R$ be any set of measure at least $\tau$, and let $V \subseteq L$ be any set of measure at least $\beta$. Then, for all $0<\nu<1$ and $\lambda_{0}=\lambda(\beta, \nu)$, we have $\operatorname{Pr}_{x \in L, y \in N(x)}[x \in V \& y \in W] \geq$ $\beta(1-\nu)\left(\tau-\lambda_{0}\right)$, where the probability is for the random experiment of first picking a random node $x \in L$ uniformly at random, and then picking a uniformly random neighbor $y$ of $x$ in the graph $G$.

The second property deals with edge-colored samplers. It basically says that removing some subset of right vertices of a sampler yields a graph which (although not necessarily bi-regular) still has the following property: Picking a random left node and then picking its random neighbor induces roughly the same distribution on the edges as picking a random right node and then its random neighbor.

Lemma 3 ([|IKWW08|). Suppose $G=G(L \cup R, E)$ is a $\lambda$-sampler, with the right degree $D$. Let $W \subseteq R$ be any subset of density at least $\tau$, and let $G^{\prime}=G\left(L \cup W, E^{\prime}\right)$ be the induced subgraph of $G$ (obtained after removing all vertices in $R \backslash W$ ), with the edge set $E^{\prime}$. Let $C o l: E^{\prime} \rightarrow\{$ red, green $\}$ be any coloring of the edges of $G^{\prime}$ such that at most $\eta D|W|$ edges are colored red, for some $0 \leq \eta \leq 1$. Then, for all $0<\nu, \beta<1$ and $\lambda_{0}=\lambda(\beta, \nu)$, we have

$$
\operatorname{Pr}_{x \in L, y \in N_{G^{\prime}}(x)}[\operatorname{Col}(\{x, y\})=r e d] \leq \max \left\{\eta /\left((1-\nu)\left(1-\lambda_{0} / \tau\right)\right), \beta\right\},
$$

where the probability is for the random experiment of first picking a uniformly random node $x \in L$, and then picking a uniformly random neighbor $y$ of $x$ in the graph $G^{\prime}$. 


\section{Dynamic Weakly Verifiable Puzzles}

We consider the following generalization of weakly verifiable puzzles (WVP) [CHS05], which we call dynamic weakly verifiable puzzles (DWVP).

Definition 1 (Dynamic Weakly Verifiable Puzzle). A DWVP $\Pi$ is defined by a distribution $\mathcal{D}$ on pairs of strings $(x, \alpha)$; here $\alpha$ is the advice used to generate and evaluate responses to the puzzle $x$. Unlike the case of WVP, here the string $x$ defines a set of puzzles, $(x, q)$ for $q \in Q$ (for some set $Q$ of indices). There is a probabilistic polynomial-time computable relation $R$ that specifies which answers are solutions for which of these puzzles: $R(\alpha, q, r)$ is true iff response $r$ is correct answer to puzzle $q$ in the collection determined by $\alpha$. Finally, there is also a probabilistic polynomial-time computable hint function $H(\alpha, q)$.

A solver can make a number of queries: query hint $(q)$ asks for $H(\alpha, q)$, the hint for puzzle number $q$; a verification query $V(q, r)$ asks whether $R(\alpha, q, r)$. The solver succeeds if it makes an accepting verification query for a $q$ where it has not previously made a hint query on $q$.

Clearly, WVP is a special case of DWVP when $|Q|=1$. A MAC is also a special case of DWVP where $\alpha$ is a secret key, $x$ is the empty string, queries $q$ are messages, a hint is to give the MAC of a message, and correctness is for a (valid message, MAC) pair. Signatures are also a special case with $\alpha$ being a secret key, $x$ a public key, and the rest similar to the case of MACs.

We give hardness amplification for such weakly verifiable puzzle collections, using direct products. First, let us define an $n$-wise direct product for DWVPs.

Definition 2 ( $n$-wise direct-product of DWVPs). Given a DWVP $\Pi$ with $\mathcal{D}, R, Q$, and $H$, its $n$-wise direct product is a DWVP $\Pi^{n}$ with the product distribution $\mathcal{D}^{n}$ producing $n$-tuples $\left(\alpha_{1}, x_{1}\right), \ldots,\left(\alpha_{n}, x_{n}\right)$. For a given $n$-tuple $\bar{\alpha}=\left(\alpha_{1}, \ldots, \alpha_{n}\right)$ and $a$ query $q \in Q$, the new hint function is $H^{n}(\bar{\alpha}, q)=\left(H\left(\alpha_{1}, q\right), \ldots, H\left(\alpha_{n}, q\right)\right)$. For $p a$ rameters $0 \leq \gamma, \delta \leq 1$, we say that the new relation $R^{k}\left(\left(\alpha_{1}, \ldots, \alpha_{n}\right), q,\left(r_{1}, \ldots, r_{n}\right)\right)$ evaluates to true if there is a subset $S \subseteq[n]$ of size at least $n-(1-\gamma) \delta n$ such that $\wedge_{i \in S} R\left(\alpha_{i}, q, r_{i}\right)$.

A solver of the $n$-wise DWVP $\Pi^{n}$ may ask hint queries hint ${ }^{n}(q)$, getting $H^{n}(\bar{\alpha}, q)$ as the answer. A verification query $V^{n}(q, \bar{r})$ asks if $R^{n}(\bar{\alpha}, q, \bar{r})$, for an $n$-tuple $\bar{r}=$ $\left(r_{1}, \ldots, r_{n}\right)$. We say that the solver succeeds if it makes an accepting verification query for a $q$ where it has not previously made a hint query on $q .1$

Theorem 3 (Security amplification for DWVP (uniform version)). Suppose a probabilistic t-time algorithm $A$ succeeds in solving the $n$-wise direct-product of some DWVP $\Pi^{n}$ with probability at least $\epsilon$, where $\epsilon \geq(800 / \gamma \delta) \cdot(h+v) \cdot e^{-\gamma^{2} \delta n / 40}$, and $h$ is the number of hint queries 2 , and $v$ the number of verification queries made by $A$.

\footnotetext{
${ }^{1}$ We don't allow the solver to make hint queries $\left(q_{1}, \ldots, q_{n}\right)$, with different $q_{i}$ 's, as this would make the new $k$-wise DWVP completely insecure. Indeed, the solver could ask cyclic shifts of the query $\left(q_{1}, \ldots, q_{n}\right)$, and thus learn the answers for $q_{1}$ in all $n$ positions, without actually making the hint query $\left(q_{1}, \ldots, q_{1}\right)$.

${ }^{2}$ Note that when $h=0$, we're in the case of WVPs.
} 
Then there is a uniform probabilistic algorithm $B$ that succeeds in solving the original DWVP $\Pi$ with probability at least $1-\delta$, while making $O((h(h+v) / \epsilon) \cdot \log (1 / \gamma \delta))$ hint queries, only one verification query, and having a running time

$$
\left.O\left((h+v)^{4} / \epsilon^{4}\right) \cdot t+(t+\omega h) \cdot(h+v) / \epsilon \cdot \log (1 / \gamma \delta)\right) .
$$

Here $\omega$ denotes the maximum time to generate a hint for a given query. The success probability of $B$ is over the random input puzzle of $\Pi$ and internal randomness of $B$.

Note that $B$ in the above theorem is a uniform algorithm. We get a reduction in running time of an algorithm for attacking $\Pi$ if we allow it to be non-uniform. The algorithm $B$ above (as we will see later in the proof) samples a suitable hash function from a family of pairwise independent hash functions and then uses the selected function in the remaining construction. In the non-uniform version of the above theorem, we can skip this step and assume that the suitable hash function is given to it as advice. Following is the non-uniform version of the above theorem.

Theorem 4 (Security amplification for DWVP (non uniform version)). Suppose a probabilistic t-time algorithm $A$ succeeds in solving the $n$-wise direct-product of some DWVP $\Pi^{n}$ with probability at least $\epsilon$, where $\epsilon \geq(800 / \gamma \delta) \cdot(h+v) \cdot e^{-\gamma^{2} \delta n / 40}, h$ is the number of hint queries, and $v$ the number of verification queries made by $A$. Then there is a probabilistic algorithm $B$ that succeeds in solving the original DWVP $\Pi$ with probability at least $1-\delta$, while making $O((h \cdot(h+v) / \epsilon)) \cdot \log (1 / \gamma \delta))$ hint queries, only one verification query, and having the running time $O((t+\omega h) \cdot((h+v) / \epsilon) \cdot \log (1 / \gamma \delta))$, where $\omega$ denotes the maximum time to generate a hint for a given query. The success probability of $B$ is over the random input puzzle of $\Pi$ and internal randomness of $B$.

\subsection{Intuition}

We want to solve a single instance of DWVP $\Pi$, using an algorithm $A$ for the $n$-wise direct-product $\Pi^{n}$, and having access to the hint-oracle and the verification-oracle for $\Pi$. The idea is to "embed" our unknown puzzle into an $n$-tuple of puzzles, by generating the $n-1$ puzzles at random by ourselves. Then we simulate algorithm $A$ on this $n$ tuple of puzzles. During this simulation, we can answer the hint queries made by $A$ by computing the hint function on our own puzzles and by making the appropriate hint query to the hint-oracle for $\Pi$. We will answer all verification queries of $A$ by 0 (meaning "failure"). At the end, we see if $A$ made a verification query which was "sufficiently" correct in the positions corresponding to our own puzzles; if so, we make a probabilistic decision to output this query (for the position of our unknown input puzzle).

To decide whether to believe or not to believe the verification query made by $A$, we count the number of correct answers it gave for the puzzles we ourselves generated (and hence can verify), and then believe with probability inverse-exponentially related to the number of incorrect answers we see (i.e., the more incorrect answers we see, the less likely we are to believe that $A$ 's verification query is correct for the unknown puzzle); since we allow up to $(1-\gamma) \delta n$ incorrect answers, we will discount these many incorrect answers, when making our probabilistic decision. 
Such a "soft" decision algorithm for testing if an $n$-tuple is good has been proposed in [IW97], and later used in [BIN97, [JK08]. Using the machinery of [IJK08], we may assume, for the sake of intuition, that we can decide if a given verification query $(q, \bar{r})$ made by $A$ is correct (i.e., is correct for at least $n-(1-\gamma) \delta n$ of $r_{i}$ 's in the $n$-tuple $\bar{r}$ ).

Since $A$ is assumed to succeed on at least $\epsilon$ fraction of $n$-tuples of puzzles, we get from $A$ a correct verification query with probability at least $\epsilon$ (for a random unknown puzzle, and random $n-1$ self-generated puzzles). Hence, we will produce a correct solution to the input puzzle of $\Pi$ with probability at least $\epsilon$.

To improve this probability, we would like to repeatedly sample $n-1$ random puzzles, simulate $A$ on the obtained $n$-tuple of puzzles (including the input puzzle in a random position), and check if $A$ produces a correct verification query. If we repeat for $O(\log 1 / \delta) / \epsilon)$ iterations, we should increase our success probability for solving $\Pi$ to $1-\delta$.

However, there is a problem with such repeated simulations of $A$ on different $n$ tuples of puzzles: in some of its later runs, $A$ may make a successful verification query for the same $q$ for which it made a hint query in an earlier run. Thus, we need to make sure that a successful verification query should not be one of the hint queries asked by $A$ in one of its previous runs. We achieve this by randomly partitioning the query space $Q$ into the "attack" queries $P$, and "hint" queries. Here $P$ is a random variable such that any query has probability $\frac{1}{2(h+v)}$ of falling inside $P$. We will define the set $P$ by picking a random hash function hash from $Q$ to $\{0,1, \ldots, 2(h+v)-1\}$, and setting $P=P_{h a s h}$ to be the preimages of 0 of hash.

We say that the first success query for $A$ is the first query where a successful verification query without a previous hint query is made. A canonical success for attacker $A$ with respect to $P$ is an attack so that the first successful verification query is in $P$ and all earlier queries (hint or verification) are not in $P$.

We will show that the expected fraction of canonical successes for $P_{\text {hash }}$ is at least $\frac{\epsilon}{4(h+v)}$. We will also give an efficient algorithm (the Pick-hash procedure below) that finds a hash function hash so that the fraction of canonical successes for $P_{\text {hash }}$ is close to the expected fraction. Then we test random candidates for being canonical successes with respect to $P_{\text {hash }}$.

Due to this extra complication (having to separate hint and verification queries), we lose on our success probability by a factor of $8(h+v)$ compared to the case of WVPs analyzed in [IJK08]. The formal proof of the theorem is given in the following subsection.

\subsection{Proof of Theorems 3 and 4}

Proof (proof of Theorem 3). For any mapping hash: $Q \rightarrow\{0, \ldots, 2(h+v)-1\}$, let $P_{\text {hash }}$ denote the preimages of 0 . Also, as defined in the previous section, a canonical success for an attacker $A$ with respect to $P \subseteq Q$ is an attack so that the first successful verification query is in $P$ and all earlier queries (hint or verification) queries are not in $P$. The proof of the main theorem follows from the following two lemmas.

Lemma 4. Let $A$ be an algorithm which succeeds in solving the $n$-wise direct-product of some DWVP $\Pi^{n}$ with probability at least $\epsilon$ while making h hint queries, $v$ verification 
queries and have a running time $t$. Then there is a probabilistic algorithm which runs in time $O\left(\left((h+v)^{4} / \epsilon^{4}\right) \cdot t\right)$ and with high probability outputs a function hash $: Q \rightarrow$ $\{0, \ldots, 2(h+v)-1\}$ such that the canonical success probability of $A$ with respect to the set $P_{\text {hash }}$ is at least $\frac{\epsilon}{8(h+v)}$.

Lemma 5. Let hash $: Q \rightarrow\{0, \ldots, 2(h+v)-1\}$ be a function. Let $A$ be an algorithm such that the canonical success probability of A over an $n$-wise DWVP $\Pi^{n}$ with respect to $P_{\text {hash }}$ is at least $\epsilon^{\prime}=(100 / \gamma \delta) \cdot e^{-\gamma^{2} \delta n / 40}$. Furthermore, let A makes h hint queries and $v$ verification queries and have a running time $t$. Then there is a probabilistic algorithm $B$ that succeeds in solving the original DWVP $\Pi$ with probability at least $1-\delta$, while making $O((h(h+v) / \epsilon) \cdot \log (1 / \gamma \delta))$ hint queries, only one verification query, and having the running time $O((t+\omega h) \cdot(h / \epsilon) \cdot \log (1 / \gamma \delta))$, where $\omega$ denotes the maximum time to generate a hint for a given query.

Proof (proof of Theorem 4). The proof follows from Lemmas 4 and 5

In the remaining subsection, we give the proof of Lemmas 4 and 5. The proof of Lemma 5 is very similar to the analysis of WVPs in [IJK08].

Proof (proof of Lemma 4). Let $\mathcal{H}$ be a pairwise independent family of hash functions mapping $Q$ into $\{0, \ldots,(2(h+v)-1)\}$. First note that for a randomly chosen function hash $\stackrel{\$}{\leftarrow}, P_{\text {hash }}$ is a random variable denoting the partition of $Q$ into two parts which satisfies the following properties:

$$
\forall q_{1}, q_{2} \in Q, \operatorname{Pr}\left[q_{1} \in P_{h a s h} \mid q_{2} \in P_{h a s h}\right]=\operatorname{Pr}\left[q_{1} \in P_{h a s h}\right]=\frac{1}{2(h+v)}
$$

For any fixed choice of $\bar{\alpha}=\left(\alpha_{1}, \ldots, \alpha_{n}\right)$, let $q_{1}^{\bar{\alpha}}, \ldots, q_{h}^{\bar{\alpha}}$ denote the hint queries of $A$ and $\left(q_{h+1}^{\bar{\alpha}}, \bar{r}_{h+1}^{\bar{\alpha}}\right), \ldots,\left(q_{h+v}^{\bar{\alpha}}, \bar{r}_{h+v}^{\bar{\alpha}}\right)$. Also, let $\left(q_{j}^{\bar{\alpha}}, \bar{r}_{j}^{\bar{\alpha}}\right)$ denote the first successful verification query, in case $A$ succeeds, and let it denote any arbitrary verification query in the case $A$ fails. Furthermore, let $E_{\bar{\alpha}}$ denote the event that $q_{1}^{\bar{\alpha}}, \ldots, q_{h}^{\bar{\alpha}}, q_{h+1}^{\bar{\alpha}}, \ldots, q_{j-1}^{\bar{\alpha}} \notin$ $P_{h a s h}$ and $q_{j}^{\bar{\alpha}} \in P_{h a s h}$. We bound the probability of the event $E_{\bar{\alpha}}$ as follows.

Claim. For each fixed $\bar{\alpha}$, we have $\operatorname{Pr}_{P_{h a s h}}\left[E_{\bar{\alpha}}\right] \geq \frac{1}{4(h+v)}$.

Proof. We have

$$
\begin{aligned}
\operatorname{Pr}_{P_{h a s h}}\left[E_{\bar{\alpha}}\right] & =\operatorname{Pr}\left[q_{j}^{\bar{\alpha}} \in P_{h a s h} \& \forall i<j, q_{i}^{\bar{\alpha}} \notin P_{h a s h}\right] \\
& =\operatorname{Pr}\left[q_{j}^{\bar{\alpha}} \in P_{h a s h}\right] \cdot \operatorname{Pr}\left[\forall i<j, q_{i}^{\bar{\alpha}} \notin P_{h a s h} \mid q_{j}^{\bar{\alpha}} \in P_{h a s h}\right] .
\end{aligned}
$$

By (11), we get that the latter expression is equal to $\frac{1}{2(h+v)} \cdot\left(1-\operatorname{Pr}\left[\exists i<j, q_{i}^{\bar{\alpha}} \in\right.\right.$ $\left.P_{\text {hash }} \mid q_{j}^{\bar{\alpha}} \in P_{h a s h}\right]$ ), which, by the union bound, is at least

$$
\frac{1}{2(h+v)} \cdot\left(1-\sum_{i<j} \operatorname{Pr}\left[q_{i}^{\bar{\alpha}} \in P_{h a s h} \mid q_{j}^{\bar{\alpha}} \in P_{h a s h}\right]\right) .
$$


Finally, using the pairwise independence property (11), we conclude that

$$
\operatorname{Pr}\left[E_{\bar{\alpha}}\right] \geq \frac{1}{2(h+v)} \cdot\left(1-\sum_{i<j} \operatorname{Pr}\left[q_{i}^{\bar{\alpha}} \in P_{h a s h}\right]\right) \geq \frac{1}{4(h+v)},
$$

as required.

Let $T$ denote the "good" set corresponding to $A$ 's attack, that is,

$T=\left\{\bar{\alpha}: A^{\prime}\right.$ s attack succeeds $\}$. We have $\operatorname{Pr}[\bar{\alpha} \in T] \geq \epsilon$. Consider the following random variable: $G_{P_{h a s h}}=\left\{\bar{\alpha} \mid \bar{\alpha} \in T\right.$ and $\left.E_{\bar{\alpha}}\right\}$. So, $G_{P_{h a s h}}$ contains those $\bar{\alpha}$ 's for which $A$ has canonical success.

Since $\forall \bar{\alpha} \in G, \operatorname{Pr}_{P_{h a s h}}\left[E_{\bar{\alpha}}\right] \geq \frac{1}{4(h+v)}$, using linearity of expectation we get $\operatorname{Exp}_{P_{h a s h}}\left[\operatorname{Pr}_{\bar{\alpha}}\left[\bar{\alpha} \in G_{P_{h a s h}}\right]\right] \geq \frac{\epsilon}{4(h+v)}$. Hence, by averaging, we get that with probability at least $\frac{\epsilon}{8(h+v)}$ over the randomness of $P_{h a s h}$, there is at least $\frac{\epsilon}{8(h+v)}$ chance that a randomly chosen $\bar{\alpha} \in G_{P_{h a s h}}$. Let us call such $P_{h a s h}$ 's "good". The subroutine Pick-hash (see figure 2) uses sampling and runs in time $O\left(\left((h+v)^{4} / \epsilon^{4}\right) \cdot t\right)$ to return a mapping hash such that $P_{\text {hash }}$ is good.

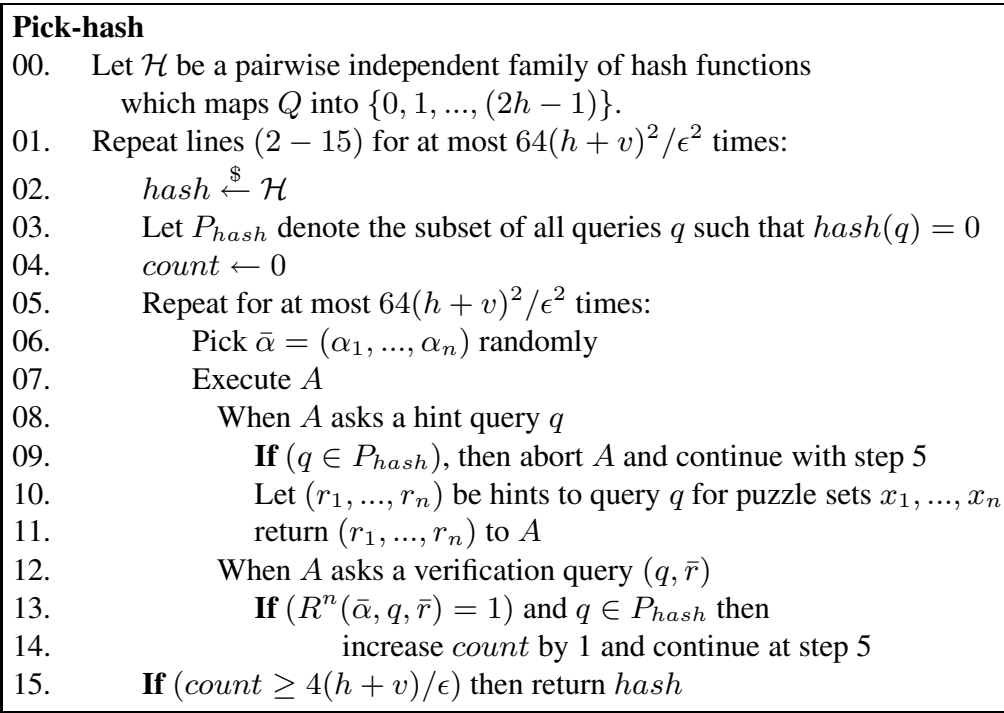

Fig. 1. Algorithm for picking a good hash function

\section{Proof (Proof of Lemma 5)}

Due to space restriction, we only give an intuitive sketch of the proof in this paper. The detailed proof of this lemma can be found in the full version of the paper. Figure (2) gives the formal description of the algorithm $B$ which uses the algorithm $A$.

For any fixed $\bar{\alpha}$, let $q_{1}^{\bar{\alpha}}, \ldots, q_{h}^{\bar{\alpha}}$ denote the hint queries made by $A$ and $\left(q_{h+1}^{\bar{\alpha}}, r_{h+1}^{\bar{\alpha}}\right), \ldots$, $\left(q_{h+v}^{\bar{\alpha}}, r_{h+v}^{\bar{\alpha}}\right)$ denote the verification queries. Let $j \in[h+v]$ be the first query such that $q_{v}^{\bar{\alpha}} \in P_{h a s h}$. For all simulations of $A, B$ correctly answers every hint query of $A$ by itself 


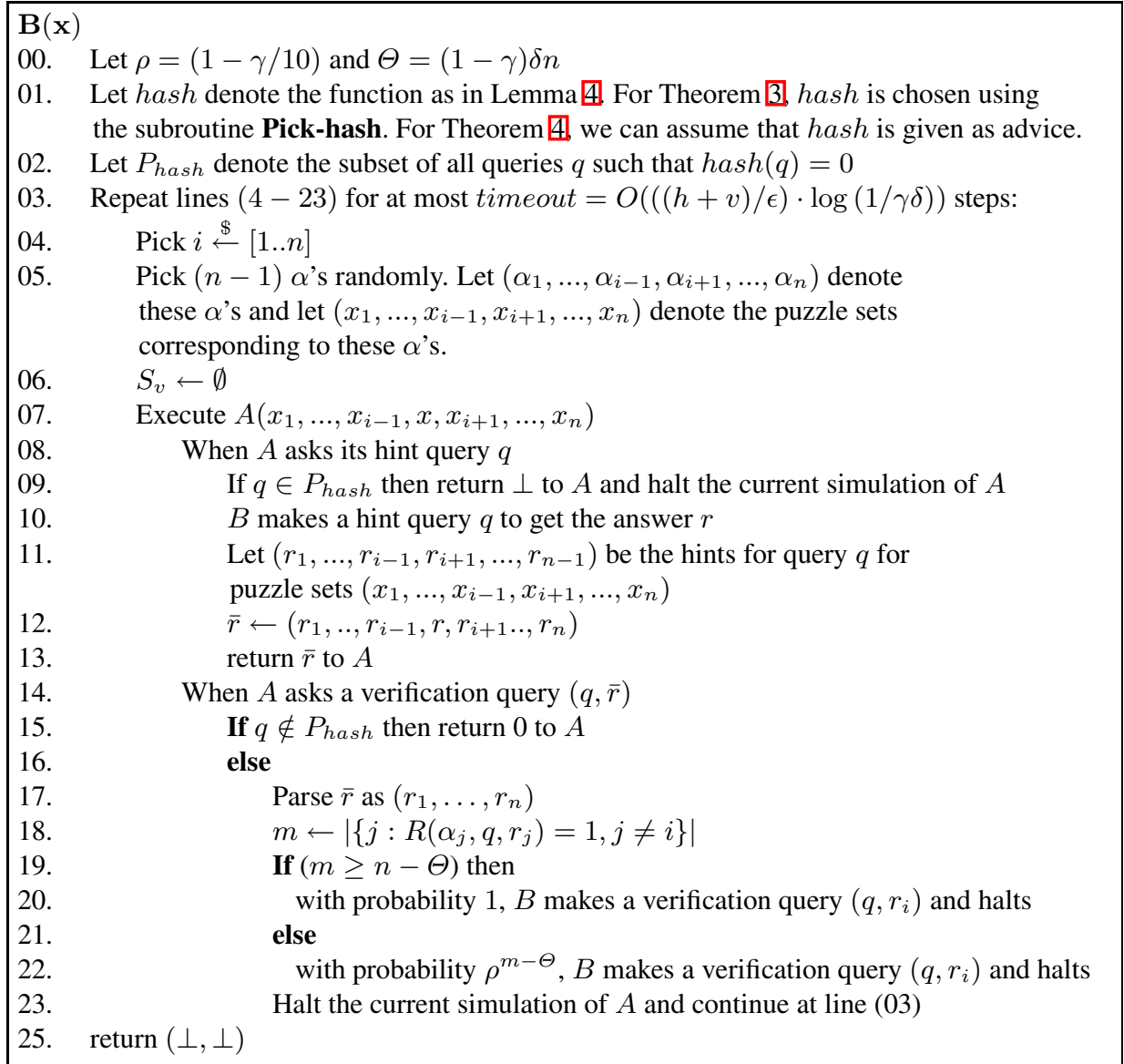

Fig. 2. Algorithm for solving $\Pi$

making a hint query with respect to the DWVP $\Pi$ which it is trying to solve (lines 10-13). Note that for a single simulation of $A$ (lines 6-25), the simulation is aborted if $j \leq h$ (line 9). Otherwise, $B$ makes a "soft" decision using $\left(q_{j}^{\bar{\alpha}}, \bar{r}_{j}^{\bar{\alpha}}\right)$ to produce its verification query (lines 19-24). Lemma 4 tells that there are at least $\epsilon^{\prime}$ fraction of $\bar{\alpha}$ 's such that $\left(q_{j}^{\bar{\alpha}}, \bar{r}_{j}^{\bar{\alpha}}\right)$ is a correct verification query for $A$ (these are $\bar{\alpha}$ 's on which $A$ has canonical success). So, intuitively there is a fair chance that $B$ produces a correct verification query.

Let Good denote the set of $\bar{\alpha}$ 's on which $A$ succeed canonically. From the assumption of the Lemma we know that Good contains at least $\epsilon^{\prime}$ fraction of $\bar{\alpha}$ 's. The remaining task is to argue that this is sufficient to show that $B$ succeeds with high probability. The rest of the analysis, apart from minor details, is similar to the proof of the Direct Product theorem for WVPs from [IJK08], essentially arguing that the lines 17-22 of the algorithm $B$ act as a decision procedure for the set Good. Next we give some details of these arguments.

Consider the following bipartite graph $G=G(L \cup R, E)$ : the set of left vertices $L$ is the set of $\alpha$ 's; the right vertices $R$ are all $n$-tuples $\bar{\alpha}=\left(\alpha_{1}, \ldots, \alpha_{n}\right)$; for every 
$y=\left(u_{1}, \ldots, u_{n}\right) \in R$, there are $n$ edges $\left(y, u_{1}\right), \ldots,\left(y, u_{n}\right) \in E$. Using Lemma we see that this graph is a $\lambda$-sampler for $\lambda(\mu, \nu)=e^{-\nu^{2} \mu k / 2}$.

For an unknown secret key $\alpha$, let $\bar{\alpha}=\left(\alpha_{1}, \ldots, \alpha_{i-1}, \alpha, \alpha_{i}, \ldots, \alpha_{n-1}\right)$ be the $n$ tuple of secret keys that corresponds to the $n$-tuple of puzzles $\left(x_{1}, \ldots, x_{n}\right)$ that $B$ will feed to $A$ in line 7 . Let $q_{1}^{\bar{\alpha}}, \ldots, q_{h}^{\bar{\alpha}}$ be the $A$ 's hint queries and $\left(q_{h+1}^{\bar{\alpha}}, \bar{r}_{h+1}^{\bar{\alpha}}\right), \ldots$, $\left(q_{h+v}^{\bar{\alpha}}, \bar{r}_{h+v}^{\bar{\alpha}}\right)$ be the verification queries. Let $j \in[h+v]$ be the first index such that $q_{j}^{\bar{\alpha}} \in P_{h a s h}$. Let $\left(q^{\bar{\alpha}}, \bar{r}^{\bar{\alpha}}\right)$ denote $\left(q_{j}^{\bar{\alpha}}, \bar{r}_{j}^{\bar{\alpha}}\right)$ in case $j>h$ and $(\perp, \perp)$ otherwise.

In the case $\left(q^{\bar{\alpha}}, \bar{r}^{\bar{\alpha}}\right) \neq(\perp, \perp), B$ makes a probabilistic decision about using $\left(q^{\bar{\alpha}}, \bar{r}^{\bar{\alpha}}\right)$ to produce its verification queries. It does that by verifying the answers to the query at all positions other than position $i$ where the unknown $\alpha$ has been planted. Let $\left(q^{\bar{\alpha}}, r^{\bar{\alpha}}\right)$ denote the verification query made by the algorithm $B$ in this simulation of $A$. If no verification query is made or if $\left(q^{\bar{\alpha}}, \bar{r}^{\bar{\alpha}}\right)=(\perp, \perp)$, then $\left(q^{\bar{\alpha}}, r^{\bar{\alpha}}\right)=(\perp, \perp)$. Let $\left(q^{B}, r^{B}\right)$ denote the single verification query made by $B$.

First we bound the probability of timeout of $B$ or in other words the probability that $\left(q^{\bar{\alpha}}, r^{\bar{\alpha}}\right)=(\perp, \perp)$ in all iterations of $B$. If $\bar{\alpha} \in$ Good, then lines 7-23 will return a verification query with probability 1 . Hence, the probability of timeout is at most the probability that $B$ never samples a neighbor $\bar{\alpha} \in \operatorname{Good}$ of $\alpha$ in the graph $G$.

Consider the set $H$ of all those left vertices $\alpha$ of $G$ such that $\alpha$ has less than $\epsilon^{\prime} / 4$ fraction of its neighbors falling into the set Good. These are precisely those $\alpha$ 's for which $B$ is likely to time out. The next lemma shows that the set $H$ is small.

Lemma 6. The set $H$ has density at most $\gamma \delta / 5$.

Proof. Suppose that the density of $H$ is greater than $\beta=\gamma \delta / 5$. Let $H^{\prime} \subseteq H$ be any subset of $H$ of density exactly $\beta$. By our assumption, we have that $\operatorname{Pr}_{\alpha \in L, w \in N(\alpha)}[\alpha \in$ $\left.H^{\prime} \& w \in G o o d\right]<\beta \epsilon^{\prime} / 4$. On the other hand, by Lemma 2 we get that the same probability is at least $\beta\left(\epsilon^{\prime}-\lambda_{0}\right) / 3$ for $\lambda_{0}=\lambda(\beta, 2 / 3)$. This is a contradiction if $\lambda_{0} \leq \epsilon^{\prime} / 4$.

Lemma 7. For every $\alpha \notin H$, we have $\operatorname{Pr}[B$ timeouts $] \leq \gamma \delta / 20$, where the probability is over the internal randomness of $B$.

Proof. By the definition of $H$, we get that the probability of timeout on any given $\alpha \notin H$ is at most $\left(1-\epsilon^{\prime} / 4\right)^{4 \ln (20 / \gamma \delta) / \epsilon^{\prime}} \leq \gamma \delta / 20$.

Next, we need to show that the probability of $R\left(\alpha, q^{B}, r^{B}\right)=0$ conditioned on the event that $\left(q^{B}, r^{B}\right) \neq(\perp, \perp)$, is small. Note that this conditional probability remains the same across all the simulations of $A$ in lines 4-23. Consider any fixed simulation of $A$ (lines 8-23) such that $\left(q^{\bar{\alpha}}, \bar{r}^{\bar{\alpha}}\right) \neq(\perp, \perp)$. Let err be the number of incorrect answers in $\bar{r}^{\bar{\alpha}}$ for the query $q^{\bar{\alpha}}$. Then if $e r r \leq(1-\gamma) \delta n$, then lines $7-25$ of $B$ produces a verification query with probability 1 . Otherwise a verification query is produced with probability that decreases exponentially (by a factor of $\rho$ ) as err increases.For this intuitive sketch of the proof, let us make a simplifying assumption there is an oracle $\mathcal{O}$ which tells whether $\bar{\alpha} \in \operatorname{Good}$ (in some sense lines 17-22 is an approximation of such an oracle). Given such an oracle, consider the the algorithm $B^{\mathcal{O}}$, which is same as $B$ except we replace lines 17-23 with the following line:

\footnotetext{
${ }^{3}$ Note that $B$ does not have access to $\alpha$ and hence does not know $\bar{\alpha}$
} 
If $\mathcal{O}$ tells that the hidden $\bar{\alpha} \in \operatorname{Good}$, then $B$ makes verification query $\left(q, r_{i}\right)$

Intuitively, lines 17-22 in $B$ is an approximation of the line above and hence $\operatorname{Pr}\left[R\left(\alpha, q^{B}, r^{B}\right)=0 \mid\left(q^{B}, r^{B}\right) \neq(\perp, \perp)\right]$ should be close to $\operatorname{Pr}\left[R\left(\alpha, q^{B^{\mathcal{O}}}, r^{B^{\mathcal{O}}}\right)=\right.$ $\left.0 \mid\left(q^{B^{\mathcal{O}}}, r^{B^{\mathcal{O}}}\right) \neq(\perp, \perp)\right]$. On the other hand, analyzing the conditional probability $\operatorname{Pr}\left[R\left(\alpha, q^{B^{\mathcal{O}}}, r^{B^{\mathcal{O}}}\right)=0 \mid\left(q^{B^{\mathcal{O}}}, r^{B^{\mathcal{O}}}\right) \neq(\perp, \perp)\right]$ is simple and is done by analyzing the following graph: Let $G^{\prime}$ be the induced subgraph of $G$ obtained after removing all vertices in $R \backslash$ Good. For each edge $\left(\left(\alpha_{1}, \ldots, \alpha_{n}\right), \alpha_{l}\right)$ of the graph $G^{\prime}$, we color this edge green if the $l^{t h}$ answer in $\bar{r}^{\bar{\alpha}}$ is correct for the query $q^{\bar{\alpha}}$, and we color it red otherwise. Consider the following random experiment $\mathcal{E}$ defined on the graph $G^{\prime}$ :

"Pick a random $\alpha \in L$, and its random incident edge $e=(\alpha, \bar{\alpha})$ in $G^{\prime}$, for $\bar{\alpha}$ containing $\alpha$ in position $l \in[n]$. If $\bar{\alpha} \in$ Good, then output $e$ with probability 1 else output $\perp$."

For each $\alpha$, we have

$$
\begin{aligned}
& \operatorname{Pr}\left[R\left(\alpha, q^{B^{\mathcal{O}}}, r^{B^{\mathcal{O}}}\right)=0 \mid\left(q^{B^{\mathcal{O}}}, r^{B^{\mathcal{O}}}\right) \neq(\perp, \perp)\right]= \\
& \quad \operatorname{Pr}[\mathcal{E} \text { outputs red edge incident to } \alpha \mid \mathcal{E} \text { outputs some edge incident to } \alpha],
\end{aligned}
$$

where the first probability is over internal randomness of $B^{\mathcal{O}}$, and the second probability is over the random choices of $\mathcal{E}$ for the fixed $\alpha$ (i.e., over the random choice of an edge $e$ incident to $\alpha$, and the random choice whether $e$ is output).

From Lemma 3 we get that:

$\operatorname{Pr}[\mathcal{E}$ outputs red edge incident to $\alpha \mid \mathcal{E}$ outputs some edge incident to $\alpha] \leq$

$$
\max \left(\frac{\eta}{(1-\nu)\left(1-\lambda_{0} / \tau\right)}, \beta\right)
$$

which is at most $\delta-\gamma \delta / 2$ for $\eta=(1-\gamma) \delta, \beta=\delta / 2, \nu=\gamma / 4$, and $\frac{\lambda_{0}}{\tau}=\frac{\lambda(\delta / 2, \gamma / 4)}{\epsilon^{\prime}} \leq$ $\gamma / 4$.

Summing up, from Lemma 6 we get that the fraction of $\alpha$ 's for which $B$ might time out is small. From Lemma 7 we get that for the remaining $\alpha$ 's, it does not time out with high probability. Furthermore, from the above argument, the conditional probability of failing to produce a correct verification query is small. Hence, the probability that $B$ fails is small.

\section{XOR Lemmas for PRGs and PRFs}

In this section, we show how to amplify security of pseudorandom (function) generators, using Direct Products (old and new) and the Goldreich-Levin decoding algorithm from Theorem [2] 


\subsection{Amplifying PRGs}

We start with PRGs. Let $G:\{0,1\}^{k} \rightarrow\{0,1\}^{\ell(k)}$ be a polynomial-time computable generator, stretching $n$-bit seeds to $\ell(k)$-bit strings, for $\ell(k)>k$, such that $G$ is $\delta(k)$ pseudorandom. That is, for any probabilistic polynomial-time algorithm $A$, and all sufficiently large $k$, we have $\left|\operatorname{Pr}_{s}[A(G(s))=1]-\operatorname{Pr}_{x}[A(x)=1]\right| \leq \delta(k)$, where $s$ is chosen uniformly at random from $\{0,1\}^{k}$, and $x$ from $\{0,1\}^{\ell(k)}$.

We say that a PRG $G$ is weak if it is $\delta$-pseudorandom for a constant $\delta<1 / 2$. We say that a PRG $G$ is strong if it is $\delta(n)$-pseudorandom for $\delta(n)<1 / k^{c}$ for any constant $c>0$ (i.e., negligible).

For the rest of this subsection, let $n>\omega(\log k)$ and let $n^{\prime}=2 n$. We show that any weak PRG $G_{\text {weak }}$ of stretch $\ell(k)>k n$ can be transformed into a strong PRG $G_{\text {strong }}$ as follows: The seed to $G_{\text {strong }}$ is a $n$-tuple of seeds to $G_{\text {weak }}$, and the output of $G_{\text {strong }}\left(s_{1}, \ldots, s_{n}\right)$ is the bit-wise XOR of the $n$ strings $G_{\text {weak }}\left(s_{1}\right), \ldots, G_{\text {weak }}\left(s_{n}\right)$.

Theorem 5 (Security amplification for PRGs). If $G_{\text {weak }}$ is a weak PRG with stretch $\ell(k)>k n$, then the generator $G_{\text {strong }}$ defined above is a strong PRG, mapping nk-bit seeds into $\ell(k)$-bit strings.

Proof. Since the proof uses standard techniques, we will only sketch it here. Let $G_{\text {weak }}$ be $\delta$-pseudorandom for $\delta<1 / 2$. The proof is by a sequence of the following steps.

1. Use Yao's "pseudorandom implies unpredictable" reduction to argue that, for a random seed $s$, each output bit $G_{\text {weak }}(s)_{i}$ (for $i \in[\ell(k)]$ ) is computable from the previous bits $G_{\text {weak }}(s)_{1 . . i-1}$ with probability at most $1 / 2+\delta$, which is some constant $\alpha<1$ since $\delta<1 / 2$ (this is where we need that $\delta<1 / 2$ ).

2. Use a Direct-Product lemma (say the one from [GNW95], or the one from the present paper, Theorem [3] to argue that, for each $i \in[\ell(k)]$, computing the direct-product $\left(G_{\text {weak }}\left(s_{1}\right)_{i}, \ldots, G_{\text {weak }}\left(s_{n^{\prime}}\right)_{i}\right)$ from $\left(G_{\text {weak }}\left(s_{1}\right)_{1 . . i-1}, \ldots, G_{\text {weak }}\left(s_{n^{\prime}}\right)_{1 . . i-1}\right)$ for independent random seeds $s_{1}, \ldots, s_{n^{\prime}}$ can't be done better than with probability $\epsilon \leq e^{-\Omega(n)}$, which is negligible.

3. Use the Goldreich-Levin decoding algorithm from Theorem 12 to argue that, for each $i \in[\ell(k)]$, computing the XOR $G_{\text {weak }}\left(s_{1}\right)_{i} \oplus \cdots \oplus G_{\text {weak }}\left(s_{n}\right)_{i}$ (i.e., $\left.G_{\text {strong }}\left(s_{1}, \ldots, s_{n}\right)_{i}\right)$ from the given bit-wise XOR of $G_{\text {weak }}\left(s_{1}\right)_{1 . . i-1}, \ldots$, $G_{\text {weak }}\left(s_{n}\right)_{1 . . i-1}$ (i.e., from $G_{\text {strong }}\left(s_{1}, \ldots, s_{n}\right)_{1 . . i-1}$ ), for independent random seeds $s_{1}, \ldots, s_{n}$, can't be done better than with probability $1 / 2+\operatorname{poly}(\epsilon n)$, which is negligibly better that random guessing.

4. Finally, using Yao's "unpredictable implies pseudorandom" reduction, conclude that $G_{\text {strong }}$ is $(\ell(k) \cdot \operatorname{poly}(\epsilon n))$-pseudorandom, which means that $G_{\text {strong }}$ is $\delta^{\prime}(k)$ pseudorandom for negligible $\delta^{\prime}(k)$, as required.

\subsection{Amplifying PRFs}

Here we would like to show similar security amplification for pseudorandom function generators (PRFs).

First we recall the definition of a PRF. Let $\left\{f_{s}\right\}_{s \in\{0,1\}^{*}}$ be a function family, where, for each $s \in\{0,1\}^{*}$, we have $f_{s}:\{0,1\}^{d(|s|)} \rightarrow\{0,1\}^{r(|s|)}$. This function family is 
called polynomial-time computable if there is polynomial-time algorithm that on inputs $s$ and $x \in\{0,1\}^{d(|s|)}$ computes $f_{s}(x)$. It is called $\delta(k)$-pseudorandom function family if, for every probabilistic polynomial-time oracle machine $M$, and all sufficiently large $k$, we have

$$
\left|\operatorname{Pr}_{s}\left[M^{f_{s}}\left(1^{k}\right)=1\right]-\operatorname{Pr}_{h_{k}}\left[M^{h_{k}}\left(1^{k}\right)=1\right]\right| \leq \delta(k),
$$

where $s$ is chosen uniformly at random from $\{0,1\}^{k}$, and $h_{k}$ is a uniformly random function from $\{0,1\}^{d(k)}$ to $\{0,1\}^{r(k)}$. Finally, we say that a PR is weak if it is $\delta$ pseudorandom for some constant $\delta<1 / 2$, and we say a PRF is strong if it is $\delta(k)$ pseudorandom for some $\delta(k)<1 / k^{c}$ for any constant $c>0$.

Let $\left\{f_{s}\right\}_{s}$ be a weak PRF. By analogy with the case of PRGs considered above, a natural idea for defining a strong PRF from $\left\{f_{s}\right\}_{s}$ is as follows: For some parameter $n$, take $n$ independent seeds $\bar{s}=\left(s_{1}, \ldots, s_{n}\right)$, and define $g_{\bar{s}}(x)$ to be the bit-wise XOR of the strings $f_{s_{1}}(x), \ldots, f_{s_{n}}(x)$.

We will argue that the defined function family $\left\{g_{\bar{s}}\right\}_{\bar{s}}$ is a strong PRF. Rather than proving this directly, we find it more convenient to prove this first for the case of weak $\operatorname{PRF}\left\{f_{s}\right\}_{s}$ of Boolean functions $f_{s}$, and use a simple reduction to get the result for general weak PRFs.

For the rest of this subsection, let $n>\omega(\log k)$ and let $n^{\prime}=2 n$.

Theorem 6 (XOR Lemma for Boolean PRFs). Let $\left\{f_{s}\right\}_{s}$ be a $\delta$-pseudorandom Boolean function family for some constant $\delta<1 / 2$. Let $\bar{s}=\left(s_{1}, \ldots, s_{n}\right)$ be a $n$ tuple of $k$-bit strings. Then, for some constant $c_{0}$ dependent on $\delta$, the following function family $\left\{g_{\bar{s}}\right\}_{\bar{s}}$ is $\epsilon$-pseudorandom for $\epsilon \leq \operatorname{poly}(k) \cdot e^{-(\delta n) / c_{0}}$ :

$$
g_{\bar{s}}(x)=f_{s_{1}}(x) \oplus \cdots \oplus f_{s_{n}}(x) .
$$

Proof. The idea is to view $\left\{f_{s}\right\}$ also as a MAC, which is a special case of a DWVP and hence we have a direct-product result (our Theorem 3 ). We will argue that if $g_{\bar{s}}$ is not a strong PRF, then one can break with non-negligible probability the direct product of MACs $\left(f_{s_{1}}, \ldots, f_{s_{n^{\prime}}}\right)$ for independent random seeds $s_{1}, \ldots, s_{n^{\prime}}$, and hence (by Theorem 3 , one can break a single $\mathrm{MAC} f_{s}$ with probability close to 1 . The latter algorithm breaking $f_{s}$ as a MAC will also be useful for breaking $f_{s}$ as a PRF, with the distinguishing probability $\delta^{\prime}>\delta$, which will contradict the assumed $\delta$-pseudorandomness of the $\operatorname{PRF}\left\{f_{s}\right\}_{s}$.

In more detail, suppose that $A$ is a polynomial-time adversary that distinguishes $g_{\bar{s}}$ from a random function, with a distinguishing probability $\epsilon>\operatorname{poly}(k) \cdot e^{-\Omega(\delta n)}$. Using a standard hybrid argument, we may assume that the first query $m$ of $A$ is decisive. That is, answering this query with $g_{\bar{s}}(m)$ and all subsequent queries $m_{i}$ with $g_{\bar{s}}\left(m_{i}\right)$ makes $A$ accept with higher probability than answering this query randomly and all subsequent queries $m_{i}$ with $g_{\bar{s}}\left(m_{i}\right)$. Let $\delta_{1}(k) \geq \epsilon /$ poly $(k)$ be the difference between the two probabilities.

Since $g_{\bar{s}}$ is a Boolean function, we can use Yao's “distinguisher-to-predictor" reduction [Yao82] to predict $g_{\bar{s}}(m)$ with probability $1 / 2+\delta_{1}(n)$ over random $n$-tuples $\bar{s}$, and for the same fixed input $m$ (since $m$ is independent from the choice of $\bar{s}$ ).

By a standard argument, we get an algorithm $A^{\prime}$ for computing the following inner product

$$
\left\langle f_{s_{1}}(m) \ldots f_{s_{n^{\prime}}}(m), z\right\rangle,
$$


for random $s_{1}, \ldots, s_{n^{\prime}}$ and a random $z \in\{0,1\}^{n^{\prime}}$, whose success probability is at least $1 / 2+\delta_{2}(k) \geq 1 / 2+\Omega\left(\delta_{1}(k) / \sqrt{n^{\prime}}\right)$; the idea is that a random $n^{\prime}=2 n$-bit string $z$ is balanced with probability $\Omega\left(1 / \sqrt{n^{\prime}}\right)$, in which case we run the predictor for $n$-XOR, and otherwise (for non-balanced $z$ ) we flip a fair random coin. Next, by averaging, we get that, for at least $\delta_{2}(k) / 2$ fraction of $n^{\prime}$-tuples $s_{1}, \ldots, s_{n^{\prime}}$, our algorithm $A^{\prime}$ correctly computes the inner product in (3) for at least $1 / 2+\delta_{2}(k) / 2$ fraction of random $z$ 's.

Applying the Goldreich-Levin algorithm from Theorem $\square$ to our algorithm $A^{\prime}$, we get an algorithm $A^{\prime \prime}$ that, for each of at least $\delta_{2}(k) / 2$ fraction of $n^{\prime}$-tuples $s_{1}, \ldots, s_{n^{\prime}}$, computes $\left(f_{s_{1}}(m), \ldots, f_{s_{n^{\prime}}}(m)\right)$ with probability at least poly $\left(\delta_{2}(k)\right)$. Hence, this algorithm $A^{\prime \prime}$ computes $\left(f_{s_{1}}(m), \ldots, f_{s_{n^{\prime}}}(m)\right)$ for a non-negligible fraction of $n^{\prime}$-tuples $s_{1}, \ldots, s_{n^{\prime}}$.

Next, we view $A^{\prime \prime}$ as an algorithm breaking the $n^{\prime}$-wise direct-product of the MAC $f_{s}$, with non-negligible probability. Using Theorem 3, we get from $A^{\prime \prime}$ an algorithm $B$ that breaks the single instance of the MAC $f_{s}$ with probability at least $1-\delta^{\prime}$ for $\delta^{\prime} \leq$ $O\left(\left(\log \left(\operatorname{poly}(k) / \delta_{2}(k)\right)\right) / n\right)$, which can be made less than $1 / 2-\delta$ for $n>\omega(\log k)$ and sufficiently large constant $c_{0}$ in the bound on $\epsilon$ in the statement of the theorem (this is where we need the assumption that $\delta<1 / 2$ ).

Note the algorithm $B$ has $1-\delta^{\prime}>1 / 2+\delta$ probability over a secret key $s$ to compute a correct message-tag pair $(\mathrm{msg}, \mathrm{tag})$ such that $f_{s}(\mathrm{msg})=\mathrm{tag}$. Also note that the algorithm $B$ makes some signing queries $f_{s}\left(q_{i}\right)=$ ? for $q_{i} \neq m s g$, but no verification queries (other than its final output pair $(m s g, t a g)$ ). We can use this algorithm $B$ to distinguish $\left\{f_{s}\right\}_{s}$ from random in the obvious way: simulate $B$ to get ( $m s g, t a g$ ) (using the oracle function to answer the signing queries of $B$ ); query the oracle function on $m s g$; if the answer is equal to tag, then accept, else reject.

Clearly, the described algorithm accepts with probability $1 / 2$ on a random oracle, and with probability greater than $1 / 2+\delta$ on a pseudorandom function $f_{s}$. This contradicts the assumption that $\left\{f_{s}\right\}_{s}$ is $\delta$-pseudorandom.

As a corollary, we get the following.

Theorem 7 (Security amplification for PRFs). Let $\left\{f_{s}\right\}_{s}$ be a weak PRF. For a parameter $n>\omega(\log k)$, take $n$ independent seeds $\bar{s}=\left(s_{1}, \ldots, s_{n}\right)$, and define $g_{\bar{s}}(x)$ to be the bit-wise XOR of the strings $f_{s_{1}}(x), \ldots, f_{s_{n}}(x)$. The obtained function family $\left\{g_{\bar{s}}\right\}_{\bar{s}}$ is a strong PRF.

Proof. Note that given a non-Boolean weak PRF $\left\{f_{s}\right\}_{s}$, we can define a Boolean function family $\left\{f_{s}^{\prime}\right\}_{s}$ where $f_{s}^{\prime}(x, i)=f_{s}(x)_{i}$, i.e., $f_{s}^{\prime}$ treats its input as an input $x$ to $f_{s}$ and an index $i \in[r(|s|)]$, and outputs the $i$ th bit of $f_{s}(x)$. Clearly, if $\left\{f_{s}\right\}_{s}$ is $\delta$ pseudorandom, then so is $\left\{f_{s}^{\prime}\right\}_{s}$.

Then we amplify the security of $\left\{f_{s}^{\prime}\right\}_{s}$, using our XOR Theorem for PRFs (Theorem 6. We obtain a strong PRF $\left\{g_{\bar{s}}^{\prime}\right\}_{\bar{s}}$, where $\bar{s}=\left(s_{1}, \ldots, s_{n}\right)$ and $g_{\bar{s}}^{\prime}(x, i)=$ $f_{s_{1}}^{\prime}(x, i) \oplus \cdots \oplus f_{s_{n}}^{\prime}(x, i)$.

Finally, we observe that our function $g_{\bar{s}}(x)$ is the concatenation of the values $g_{\bar{s}}^{\prime}(x, i)$ for all $1 \leq i \leq r(|k|)$. This function family $\left\{g_{\bar{s}}\right\}_{\bar{s}}$ is still a strong PRF, since we can simulate each oracle access to $g_{\bar{s}}$ with $d(|s|)$ oracle calls to $g_{\bar{s}}^{\prime}$. 


\section{Conclusions}

We have established security amplification theorems for several interactive cryptographic primitives, including message authentication codes, digital signature and pseudorandom functions. The security amplifications for MACs and SIGs follow the direct product approach and work even for the weak variants of these primitives with imperfect completeness. For $\delta$-pseudorandom PRFs, we have shown that the standard XOR lemma works for any $\delta<\frac{1}{2}$, which is optimal, complementing the non-standard XOR lemma of [Mye03], which works even for $\frac{1}{2} \leq \delta<1$.

Of independent interest, we abstracted away the notion of dynamic weakly verifiable puzzles (DWVPs), which generalize a variety of known primitives, including ordinary WVPs, MACs and SIGs. We have also shown a very strong Chernoff-type security amplification theorem for DWVPs, and used it to establish our security amplification results for MACs, SIGs and PRFs.

Acknowledgments. Yevgeniy Dodis was supported in part by NSF Grants 0831299, 0716690, 0515121, 0133806. Part of this work was done while the author was visiting the Center for Research on Computation and Society at Harvard University. Russell Impagliazzo was supported in part NSF Grants $0716790,0835373,0832797$, and by the Ellentuck Foundation. Ragesh Jaiswal was supported in part by NSF Grant 0716790, and completed part of this work while being at the University of California at San Diego.

\section{References}

[BIN97] Bellare, M., Impagliazzo, R., Naor, M.: Does parallel repetition lower the error in computationally sound protocols? In: Proceedings of the Thirty-Eighth Annual IEEE Symposium on Foundations of Computer Science, pp. 374-383 (1997)

[CHS05] Canetti, R., Halevi, S., Steiner, M.: Hardness amplification of weakly verifiable puzzles. In: Kilian, J. (ed.) TCC 2005. LNCS, vol. 3378, pp. 17-33. Springer, Heidelberg (2005)

[Cor00] Coron, J.S.: On the exact security of full domain hash. In: Bellare, M. (ed.) CRYPTO 2000. LNCS, vol. 1880, pp. 229-235. Springer, Heidelberg (2000)

[CRS ${ }^{+}$07] Canetti, R., Rivest, R., Sudan, M., Trevisan, L., Vadhan, S., Wee, H.: Amplifying collision resistance: A complexity-theoretic treatment. In: Menezes, A. (ed.) CRYPTO 2007. LNCS, vol. 4622, pp. 264-283. Springer, Heidelberg (2007)

[DNR04] Dwork, C., Naor, M., Reingold, O.: Immunizing encryption schemes from decryption errors. In: Cachin, C., Camenisch, J.L. (eds.) EUROCRYPT 2004. LNCS, vol. 3027, pp. 342-360. Springer, Heidelberg (2004)

[GL89] Goldreich, O., Levin, L.A.: A hard-core predicate for all one-way functions. In: Proceedings of the Twenty-First Annual ACM Symposium on Theory of Computing, pp. 25-32 (1989)

[GNW95] Goldreich, O., Nisan, N., Wigderson, A.: On Yao's XOR-Lemma. Electronic Colloquium on Computational Complexity, TR95-050 (1995)

[Gol01] Goldreich, O.: Foundations of Cryptography: Basic Tools. Cambridge University Press, New York (2001)

[IJK08] Impagliazzo, R., Jaiswal, R., Kabanets, V.: Chernoff-type direct product theorems. Journal of Cryptology (published online September 2008); preliminary version in CRYPTO 2007 
[IJKW08] Impagliazzo, R., Jaiswal, R., Kabanets, V., Wigderson, A.: Uniform direct-product theorems: Simplified, optimized, and derandomized. In: Proceedings of the Fortieth Annual ACM Symposium on Theory of Computing, pp. 579-588 (2008)

[Imp95] Impagliazzo, R.: Hard-core distributions for somewhat hard problems. In: Proceedings of the Thirty-Sixth Annual IEEE Symposium on Foundations of Computer Science, pp. 538-545 (1995)

[IW97] Impagliazzo, R., Wigderson, A.: P=BPP if E requires exponential circuits: Derandomizing the XOR Lemma. In: Proceedings of the Twenty-Ninth Annual ACM Symposium on Theory of Computing, pp. 220-229 (1997)

[Lev87] Levin, L.A.: One-way functions and pseudorandom generators. Combinatorica 7(4), 357-363 (1987)

[LR86] Luby, M., Rackoff, C.: Pseudorandom permutation generators and cryptographic composition. In: Proceedings of the Eighteenth Annual ACM Symposium on Theory of Computing, pp. 356-363 (1986)

[Mye03] Myers, S.: Efficient Amplification of the Security of Weak Pseudo-Random Function Generators. J. Cryptology 16(1), 1-24 (2003)

[Mye99] Myers, S.: On the development of block-ciphers and pseudorandom function generators using the composition and XOR operators. Master's thesis, University of Toronto (1999)

[NR98] Naor, M., Reingold, O.: From unpredictability to indistinguishability: A simple construction of pseudo-random functions from MACs. In: Krawczyk, H. (ed.) CRYPTO 1998. LNCS, vol. 1462, pp. 267-282. Springer, Heidelberg (1998)

[NR99] Naor, M., Reingold, O.: On the construction of pseudorandom permutations: Luby-Rackoff revisited. Journal of Cryptology, 29-66 (1999)

[PV07] Pass, R., Venkitasubramaniam, M.: An efficient parallel repetition theorem for Arthur-Merlin games. In: Proceedings of the Thirty-Ninth Annual ACM Symposium on Theory of Computing, pp. 420-429 (2007)

[PW07] Pietrzak, K., Wikstrom, D.: Parallel repetition of computationally sound protocols revisited. In: Vadhan, S.P. (ed.) TCC 2007. LNCS, vol. 4392, pp. 86-102. Springer, Heidelberg (2007)

[Yao82] Yao, A.C.: Theory and applications of trapdoor functions. In: Proceedings of the Twenty-Third Annual IEEE Symposium on Foundations of Computer Science, pp. 80-91 (1982) 